\title{
Determining the Pregnancy Status of Patients Before Diagnostic Nuclear Medicine Procedures: The Australian Experience
}

\author{
Daphne J. James ${ }^{1}$, Paul Cardew ${ }^{1,2}$, and Helen M. Warren-Forward ${ }^{1}$ \\ ${ }^{I}$ School of Health Sciences, University of Newcastle, Newcastle, Australia; and ${ }^{2}$ Hunter New England Imaging, Hunter New England \\ Area Health Service, Newcastle, Australia
}

\begin{abstract}
lonizing radiation used in diagnostic nuclear medicine procedures has the potential to have biologic effects on a fetus. Nuclear medicine technologists (NMTs) therefore have a responsibility to ensure that they question all patients of childbearing age about their pregnancy status before starting any procedure, to avoid unnecessary fetal irradiation. In Australia, there are no clearly defined practice guidelines to assist NMTs in determining whom to question or how to question their patients. Methods: Semistructured interviews were conducted with chief NMTs and staff NMTs in 8 nuclear medicine departments in Australia. Questions were based around 5 areas: regulations and policy, fetal radiation exposure, questioning of the patient, difficulties in determining pregnancy status, and the impact of the use of hybrid imaging. Audio files of the interviews were transcribed and coded. Results: Topics were coded into 5 themes: policy and awareness of guidelines, questioning the patient, radiation knowledge, decisions and assumptions made by NMTs, and the use of pregnancy testing. There was a wide variation in practice between and within departments. NMTs demonstrated a lack of knowledge and awareness of the possible biologic effects of radiation. Conclusion: This study identified a need in Australia for nuclear medicine to arrive at a consensus approach to verifying a patient's pregnancy status so that NMTs can successfully question patients about their pregnancy status. Continuing education programs are also required to keep NMTs up to date in their knowledge.
\end{abstract}

Key Words: ionizing radiation; pregnancy status; fetal exposure; qualitative

J Nucl Med Technol 2011; 39:220-225

DOI: 10.2967/jnmt.111.088740

D iagnostic nuclear medicine procedures involve the administration of radioactive materials, usually intravenously. These radiopharmaceuticals emit ionizing radiation, which

\footnotetext{
Received Jan. 31, 2011; revision accepted Mar. 29, 2011.

For correspondence or reprints contact: Daphne J. James, School of Health Sciences, University of Newcastle, University Dr., Callaghan, NSW 2308, Australia,

E-mail: Daphne.James@newcastle.edu.au

Published online Jul. 27, 2011.

COPYRIGHT @ 2011 by the Society of Nuclear Medicine, Inc.
}

has the potential to have biologic effects on humans. The injected radiopharmaceutical circulates within the bloodstream of the patient and irradiates the entire body. In Australia, nuclear medicine technologists (NMTs) administer the radiopharmaceutical for most nuclear medicine procedures. Although the radiation dose from these procedures is relatively small, the potential for biologic effects on injected patients is recognized ( 1 ).

Care must be taken not to administer radiopharmaceuticals to a patient who is pregnant, as biologic damage to the developing fetus may result (2). The response after exposure to ionizing radiation depends on several factors, including total dose, dose rate, radiation quality, and the stage of fetal development at the time of exposure; together, these factors determine the type and extent of damage that may occur (3). Developmental consequences can be teratogenic, mutagenic, or carcinogenic (3). The most radiosensitive period for the fetus is during organogenesis, which occurs at weeks 2-8 after conception $(4,5)$. At this early stage of the pregnancy, many patients are unaware that they are pregnant, and therefore, to protect the developing fetus, it is important that the NMTs have clear guidelines for ascertaining the pregnancy status of their patients. Several well-recognized published documents provide guidance on the radiologic imaging of pregnant patients. These include documents from international and national organizations such as the International Commission on Radiation Protection (2), the Society of Nuclear Medicine (6), and the Australian Radiation Protection and Nuclear Safety Agency (ARPANSA) (7).

In 2008, ARPANSA published the Safety Guide for Radiation Protection in Nuclear Medicine (7), which provides advice and guidance on radiation practice. Section 5 discusses the protection of the embryo or fetus. The guide states that "illustrated signs" advising patients to inform staff if they may be pregnant are to be placed in prominent places within a nuclear medicine department. The guide also states that "staff have a responsibility to enquire about the possibility of pregnancy in all female patients of childbearing age." ARPANSA recommends that the patient be given an explanation of why the question is being asked to ensure full cooperation and a truthful response. The guide 
identifies this questioning as a sensitive issue that requires "tact and discretion," especially with teenagers and if language barriers exist. When pregnancy status is deemed uncertain, ARPANSA recommends that the nuclear medicine physician be consulted to decide whether to postpone the procedure or perform a pregnancy test.

All professional and regulatory bodies governing the use of ionizing radiation for diagnostic imaging procedures recommend verification of the pregnancy status of all female patients of childbearing age before any procedure using ionizing radiation (7). It is thus the NMT's responsibility to perform this verification; however, the documents contain no clear guidelines on what constitutes childbearing age or how to determine the patient's pregnancy status.

All NMTs are trained to question their female patients, but it became evident from talking to NMT colleagues around Australia that the methods used vary greatly between departments and even between staff members within a department. Typically, the NMT will verbally question the patient immediately before administering the radiopharmaceutical. Some departments ask patients to sign a form stating they are not pregnant and may also require them to provide the date of their last menstrual period. Urine and serum pregnancy tests can be used to determine whether a patient is pregnant, but they are not routinely applied.

In recent years, hybrid imaging systems such as SPECT/ $\mathrm{CT}$ and PET/CT have been introduced and rapidly incorporated into general nuclear medicine practice in Australia. The use of CT combined with SPECT or PET can increase the patient's radiation exposure and hence the dose to the fetus by combining the exposure from the radiopharmaceutical with that from CT. Depending on the CT exposure factors used, "the effective dose to the patient from the CT component may be larger than that of the radiopharmaceutical" (7). If the CT is performed over the abdomen or pelvis of a pregnant patient, there will be an increased risk to the fetus.

In 2007, Applegate (8) suggested that the American College of Radiology develop a national guideline on screening patients for pregnancy before diagnostic radiology procedures to provide a standardized approach. The article highlighted the lack of any survey data investigating current practice and an apparent wide variation in departmental procedures. Topics suggested for the proposed guidelines included how the patient should be questioned, the age range for screening, the use of urine and blood pregnancy testing, and documentation. In 2008, the American College of Radiology released the ACR Practice Guideline for Imaging Pregnant or Potentially Pregnant Adolescents and Women with Ionizing Radiation (9). This guideline addresses the possible radiation risks to the fetus and how to screen for pregnancy. The guideline was developed for diagnostic radiology and specifically states that "it does not address issues for nuclear medicine."

In 2009, Schreiner-Karoussou (10) published a preliminary review of European practice concerning ionizing radiation and pregnancy that concluded there was "no harmonisation on this issue at the European level." The report suggested that there was a lack of consistent practice and thinking in this area among health professionals and that more research was required to give it the merit it deserves.

Our paper describes the findings from a qualitative study investigating current practice in Australia for determining the pregnancy status of a patient before diagnostic nuclear medicine procedures.

\section{MATERIALS AND METHODS}

Ethics approval for the study was granted by the University of Newcastle Human Research Ethics Committee in September 2009 (approval H-2009-0270).

After a literature review, interview questions were developed to investigate current departmental policies and practice, NMT knowledge of the biologic effects of radiation and fetal exposure, and problems NMTs associate with determining a patient's pregnancy status. These questions formed the basis of semistructured interviews conducted with chief NMTs and members of their staff.

Eighteen nuclear medicine departments were invited to participate. These included departments from each state of Australia and covered a variety of metropolitan and rural, and public and private, centers. A package sent to the chief NMT of each department contained an information sheet and consent form for the chief NMT, as well as several letters to be distributed among the NMTs working in the department. These letters contained an information sheet, a consent form, and a short demographic questionnaire. If the NMTs wished to participate, they were asked to return the consent form and questionnaire to us. Interviews were conducted at a time and place convenient to the participants.

A guidebook was used during all interviews to ensure that each interviewee was asked similar questions on a series of themes: regulations and policy, fetal radiation exposure, questioning of the patient, difficulties in determining pregnancy status, and impact of the use of hybrid imaging.

All interviews were recorded using a DS-50 digital voice recorder (Olympus). The audio files were transcribed using an online service, and the identity of each participant was masked during transcription. The transcripts were returned to interviewees for review and editing before analysis. After review, each transcript was printed and a paper copy stored for review and analysis. Initial topic coding was performed on the paper transcripts, and computer coding was performed using NVivo software (version 8.0; QSR International).

\section{RESULTS}

A total of 16 interviews were conducted from March to October 2010 with 8 nuclear medicine departments. Four departments were within public hospitals, and 4 were private practices. All staff NMTs had a minimum of $3 \mathrm{y}$ of experience working as an Australian and New Zealand Society of Nuclear Medicine-accredited NMT. Table 1 summarizes the demographic information for all departments and participants.

Determining Pregnancy Status - James et al. 
TABLE 1

Participant Information

\begin{tabular}{|c|c|c|c|c|c|c|c|}
\hline Department & Australian state & $\begin{array}{c}\text { Public or } \\
\text { private }\end{array}$ & $\begin{array}{c}\text { Participant } \\
\text { ID }\end{array}$ & Sex & $\begin{array}{c}\text { Years } \\
\text { as NMT }\end{array}$ & $\begin{array}{c}\text { NMT } \\
\text { position }\end{array}$ & Method of questioning \\
\hline \multirow[t]{2}{*}{1} & \multirow[t]{2}{*}{ Australian Capital Territory } & \multirow[t]{2}{*}{ Private } & C1 & M & $>5$ & Chief & Verbal and signature \\
\hline & & & $\mathrm{S} 1$ & $M$ & $>2$ & Staff & Verbal and signature \\
\hline \multirow[t]{2}{*}{2} & \multirow[t]{2}{*}{ Queensland } & \multirow[t]{2}{*}{ Private } & $\mathrm{C} 2$ & M & $>25$ & Chief & Verbal and signature \\
\hline & & & S2 & $\mathrm{F}$ & $>2$ & Staff & Verbal and signature \\
\hline \multirow[t]{2}{*}{3} & \multirow[t]{2}{*}{ New South Wales } & \multirow[t]{2}{*}{ Private } & C3 & $\mathrm{F}$ & $>15$ & Chief & Verbal only \\
\hline & & & S3 & $\mathrm{F}$ & $>2$ & Staff & Verbal only \\
\hline \multirow[t]{2}{*}{4} & \multirow[t]{2}{*}{ Western Australia } & \multirow[t]{2}{*}{ Private } & C4 & $\mathrm{F}$ & $>5$ & Chief & Verbal only \\
\hline & & & S4 & $\mathrm{F}$ & $>10$ & Staff & Verbal only \\
\hline \multirow[t]{2}{*}{5} & \multirow[t]{2}{*}{ Australian Capital Territory } & \multirow[t]{2}{*}{ Public } & C5 & M & $>25$ & Chief & Verbal and signature \\
\hline & & & S5 & $\mathrm{F}$ & $>5$ & Staff & Verbal and signature \\
\hline \multirow[t]{2}{*}{6} & \multirow[t]{2}{*}{ Victoria } & \multirow[t]{2}{*}{ Public } & C6 & M & $>25$ & Chief & Verbal, LMP, and signature \\
\hline & & & S6 & $\mathrm{F}$ & $>2$ & Staff & Verbal, LMP, and signature \\
\hline \multirow[t]{2}{*}{7} & \multirow[t]{2}{*}{ Queensland } & \multirow[t]{2}{*}{ Public } & C7 & $\mathrm{M}$ & $>10$ & Chief & Written form and signature \\
\hline & & & S7 & $M$ & $>2$ & Staff & Written form and signature \\
\hline \multirow[t]{2}{*}{8} & \multirow[t]{2}{*}{ Western Australia } & \multirow[t]{2}{*}{ Public } & $\mathrm{C} 8$ & M & $>25$ & Chief & Verbal, LMP, and signature \\
\hline & & & S8 & $\mathrm{F}$ & $>10$ & Staff & Verbal, LMP, and signature \\
\hline
\end{tabular}

$\mathrm{LMP}=$ last menstrual period.

Topic coding identified 37 free nodes (topics), which were further categorized into 5 tree nodes (themes). The themes were policy and awareness of guidelines, questioning the patient, radiation knowledge, decisions and assumptions made by NMTs, and use of pregnancy testing.

Three of these themes were aligned with those used at the interview, and the additional themes-decisions and assumptions made by NMTs and the use of pregnancy testing - were related to the question theme of difficulties in determining pregnancy status. The impact of the use of hybrid imaging was included in the theme of "radiation knowledge."

\section{Policy and Awareness of Guidelines}

All interviewees were asked if they knew whether their department had a written policy on verifying the patient's pregnancy status. Only 1 participant (C6) said his department had a written policy but it was "not that readily available" to the NMT working in the department. Nine participants thought that their procedure protocol documents, radiation safety manuals, or consent forms would have information on this.

"In our protocol manual and I think there is also a blurb written with the Radiation Safety Manual as well." (S1)

"We don't have a written policy as such however it is written into all of our consent forms." (C7)

Fifteen of the 16 participants (94\%) were not aware of any guidelines or policy statements from the Australian and New Zealand Society of Nuclear Medicine, ARPANSA, or other professional bodies that dealt specifically with how to verify a patient's pregnancy status.

\section{Questioning the Patient}

Australian policy and guidelines referring to the use of ionizing radiation in medical imaging state that all female patients of childbearing age must be questioned about their pregnancy status. However "childbearing age" does not have a clearly defined range. Participants were asked what age range of patients they questioned and how they determined which patients would be considered within childbearing age.

There were varied responses from all departments and between the chief and staff NMTs of each department. In 6 of the 8 departments, the responses from the chief and staff NMTs differed. Eight (50\%) NMTs specified age ranges from 12,14 , or $16 \mathrm{y}$ to $50-55 \mathrm{y}$. Eight participants (50\%) stated that an age range was not specified in their department, and thus, each NMT made the decision on which patients to question.

"I don't believe we have guidelines for the age. That's just up to the tech at the time." (C2)

"Pretty much from 16 to 50, but anything under that, and depending where you work, we have a lower class area around us that-you have your suspicions when a young girl comes through, we usually ask the parent to leave the room, and we will question them under the age of 15, 16, if we feel it's necessary" (S6)

"There's a lot of people doing IVF and things these days and having children in their 40s and even into their late 40s sort of thing." (C6)

Interviewees were asked to describe how they questioned their female patients about pregnancy status. Fourteen participants $(87.5 \%)$ said that they verbally questioned the patient, and 2 participants $(12.5 \%)$ said they used a written form that the patient completed and signed. Twelve participants said they required the patient to sign or initial some type of document that indicated she was not pregnant at the time of administration of the radiopharmaceutical. This was stored as part of the patient referral documents. Only verbal 
questioning was used by 4 participants, who all worked in private practice. Only 4 participants routinely asked the patient when her menstrual period was. There was $100 \%$ agreement between the answers of the chief NMT and the staff NMTs in all departments. The method of questioning used in each department is displayed in Table 1.

All participants using verbal questioning, with or without a signed document, were doubtful that there was any legal validity to this method if challenged in a court of law. Verbal questioning alone left the NMT open to a possible "he said, she said" scenario. According to ARPANSA (7), the "ultimate decision to perform or reject each individual nuclear medicine procedure" lies with the nuclear medicine specialist (the radiation medical practitioner). The referring doctor also has a responsibility to alert the nuclear medicine specialist if a patient may be pregnant.

The participants in the study felt that when the patient gave her signature or initials, it was more a case of the patient agreeing to document that she had been asked about her pregnancy status rather than declaring that she was not pregnant.

"I guess we think it adds, like I say, more the evidence that we've asked the question because if someone says I don't remember that question or we've got at least proof that we did ask it and they must have read-it's a simple statement. So it's more that that gives us confidence that we've checked. Whether it will help our case further in a legal setting, I think we would have to wait and see." (C5)

In most instances the patient would be asked "Is there any chance you could be pregnant?" or something similar. If the patient responded "No" and was "100\% sure," the NMT would usually go ahead with the procedure with no further questioning. Only when the patient seemed unsure or said she was trying to conceive did the NMT consider asking questions about her last menstrual period, contraceptive methods, or sexual activity.

Several types of patients were identified as potentially problematic. Teenagers were the main group discussed, with most NMTs stating it was difficult not only to decide which young girls to question but also to get a reliable, truthful answer from a girl, especially if she was accompanied by her parents. In some departments, they attempt to take the girl to a private area without the parent and question her there.

"The difficulties obviously are young females with parents in attendance. If they're around the age of 13/14 you try to separate them from the parents so you can actually get them to answer truthfully." (C2)

"If the father is standing right next to you when you're asking quite a young girl, I find that quite difficult. I also find it difficult because it's very uncomfortable for the young girl as well because the father's standing there looking at them going, I'm hoping you're not sexually active. But I do find that quite difficult." (S3)

Other patients identified as potentially difficult were those with language barriers, different cultural backgrounds, or mental disability and inpatients on certain medications. With these patients, the NMT was not always sure the patient understood the question being asked or the reasons for it.

"The other group I guess would be the ones who come in who don't speak English, maybe from a different cultural background" (C7)

"I suppose if you've got a patient of childbearing age who's got any sort of mental or-mental retardationthey're always difficult to ascertain. Just because they've got a disability, you can't rule them out from being pregnant." (C2)

"We have so many inpatients we get a lot of people that are on morphine and all sorts of analgesia and are not aware that they've actually signed something, or that we've given them something or why we've given it to them, even though we have described why." (S5)

\section{Radiation Knowledge}

The interviews included direct questions aimed at ascertaining the NMT's knowledge of, and attitudes toward, ionizing radiation and in particular fetal radiation exposure. Most participants $(81 \%)$ thought that the most radiosensitive time during gestation was in the first trimester, and only 1 participant could narrow that down to a more discrete time frame. When questioned about the possible consequences of fetal irradiation, only 1 NMT (S8) could give specific information on the biologic effects that may occur. Four participants said they did not know the possible consequences, and 11 participants gave vague responses.

"I guess it could lead to some sort of congenital defect." (C1)

"Deformities in the fetus and developmental problems and stuff like that." (S2)

"I guess there would be increased chances of childhood cancers and whatnot like that, but outside of that I don't know." (S7)

The NMTs were asked whether they would be concerned if they or their partner were irradiated while unknowingly pregnant. Eight participants $(50 \%)$ said they would be concerned, and 1 of these said they would terminate the pregnancy. The reasons given for their concern were the same as the reasons the other NMTs gave for not being concerned. Almost all of them said that their knowledge of radiation was sufficient to make them believe that either the fetus would be safe, or that possible biologic damage could ensue.

"I'd be concerned. It's the sort of thing that because you sort of work in radiation safety and radiation you're a bit more sort of switched on about it." (S1)

"I don't think I would, and I don't know whether that meant I'm too blasé about radiation or just informed enough to not be concerned about the risk." (C6)

\section{Decisions and Assumptions Made by NMTs}

There were many comments suggesting that NMTs rely on the patient's word when she says that she is not pregnant. The NMT has a professional responsibility to question the patient about her pregnancy status; however, the patient also has a responsibility to answer truthfully to the best of 
her knowledge. If a patient confidently answers that she does not think she could be pregnant, most NMTs said they would accept that response and proceed with the procedure without any further questioning.

"It's not a direct written formal questionnaire; it's purely just based on someone's word." (S1)

"As a rule the patient's word is usually enough and then they sign that form." (C6)

\section{Use of Pregnancy Testing}

The routine use of serum pregnancy testing on all female patients would be time-consuming, expensive, and impractical. Patients would have to be sent to a pathology service to have blood withdrawn. In a hospital department that provides this service, obtaining a result could take approximately $1 \mathrm{~h}$. This delay would be inconvenient for the patient and disrupt department workflow schedules. Urine pregnancy tests are a quick way to check if a patient is pregnant, but the results may be unreliable if performed before the date of missed menses and hence are appropriate in only certain cases (11).

Participants were asked whether they used pregnancy testing, under which circumstances they tested, and which types of tests they used. All NMTs except 1 said they had used pregnancy tests but not routinely. Most used the tests when a patient expressed uncertainty about her pregnancy status. Seven participants said they had used only serum $\beta$-human chorionic gonadotropin (hCG) tests, whereas 6 used only urine tests. One participant could not recall ever using a pregnancy test on a patient. In 3 departments, the chief and staff NMTs gave differing answers as to the type of testing they used.

\section{DISCUSSION}

Nuclear medicine procedures use ionizing radiation, which potentially can have a biologic effect on a fetus. The various organizations that provide radiation protection information and guidance recommend, but do not regulate, how NMTs question female patients of childbearing age about their pregnancy status. This preliminary study investigating NMT practice regarding pregnancy status has highlighted the need for reeducation and an Australia-wide consensus approach to questioning female patients.

This study has shown that a variety of methods of questioning is used across Australia and that NMTs show a lack of awareness of departmental and national policy. Verbal questioning is still widely used but is not usually documented and hence may lead to possible legal complications in the event a patient is irradiated in the early phases of pregnancy.

Although it is recommended that all female patients of childbearing age be questioned, this age range has not been clearly defined. There appears to be no set age limits to which NMTs adhere, and the lower age limit varies within and between departments. A patient from 12 to $16 \mathrm{y}$ old may be questioned, depending on the NMT's assessment of the patient. Women delaying pregnancy until later in life and the increasing number of older women having in vitro fertilization pregnancies are seen as a reason to ask women up until the age of 55 or $60 \mathrm{y}$. However, it may be more prudent to question patients about the date of their last menstrual period. This method would identify younger patients who have not begun menstruation and older patients who have completed menopause.

NMTs identified teenagers as one of the most difficult patient groups to question about pregnancy status. The NMTs require tact and sensitivity to ensure a truthful response to questions about pregnancy, especially if the parents are present. Removing the girl to another area, often under the guise of weighing her, is a common tactic used to allow the question to be asked in private without the parent. NMTs in this study reported using visual assessment of the patient and their own discretion to decide which young girls to question. Teenage pregnancy rates are declining across the industrialized world (12). However, both the age at menarche and the age of first sexual experience are also reported to be declining (13), making it imperative for NMTs to ensure that all their younger patients are adequately informed of the risks of radiation and questioned about possible pregnancy.

In Australia, NMTs are required to complete radiation protection and radiation biology education as part of their training courses (14). This study identified a lack of knowledge of the possible biologic effects of fetal irradiation. The fact that all participants had completed their training more than $2 \mathrm{y}$ before the study indicates a need for emphasis on ongoing education in this area.

Pregnancy testing is not routinely used in Australia to screen for pregnancy before diagnostic imaging. hCG is produced after implantation of the conceptus. Detecting hCG levels in early pregnancy is made difficult by variability in the timing of implantation (6-12 d after ovulation) and in the timing of ovulation after the onset of the last menstrual period (11). Urine pregnancy test kits are widely available and relatively inexpensive. However, they have a high rate of false-negative results when used before the date of missed menses $(15,16)$ and hence may fail to identify a pregnant patient if the test is performed too early. Serum hCG tests are able to detect smaller concentrations of hCG than urine tests (17) and therefore are more accurate when used before the date of missed menses. Nuclear medicine practices should carefully assess their use of pregnancy testing to ensure patients in the early stages of pregnancy are identified before they undergo a procedure using ionizing radiation.

\section{CONCLUSION}

There appears to be a wide variation in the approaches NMTs use to determine a patient's pregnancy status in nuclear medicine departments in Australia. Verbal questioning is the most common approach used; with or without the addition of a patient's signature to document her response. The age range for childbearing needs to be clearly defined, and NMTs should ensure that all patients within this range 
are questioned. A surprising finding of the study was that NMTs often visually assess patients and use their own discretion when deciding whom to question. NMTs also place a great deal of reliance on the patient's word when she says she is not pregnant. The use of pregnancy testing before the date of missed menses should be carefully assessed to determine the most accurate test to detect early pregnancy. This study has identified a lack of a consistent approach by NMTs in Australia when questioning female patients about their pregnancy status before diagnostic nuclear medicine procedures. There is a need for reeducation and a consensus approach to ensure that pregnant patients are not irradiated unnecessarily.

\section{ACKNOWLEDGMENT}

Funding for transcription of the audio files and for travel expenses associated with the interviews was provided by University of Newcastle New Staff Grant G0190283. No other potential conflict of interest relevant to this article was reported.

\section{REFERENCES}

1. UNSCEAR 2008 Report: Volume II-Sources and Effects of Ionizing Radiation. New York, NY: United Nations; 2008.
2. Pregnancy and medical radiation: ICRP publication 84. Ann ICRP. 2000;30: $1-44$.

3. De Santis M, Cesari E, Nobili E, Straface G, Cavaliere AF, Caruso A. Radiation effects on development. Birth Defects Res C Embryo Today. 2007;81:177-182.

4. Cousins C. Medical radiation and pregnancy. Health Phys. 2008;95:551-553.

5. De Santis M, Di Gianantonio E, Straface G, et al. Ionizing radiations in pregnancy and teratogenesis: a review of literature. Reprod Toxicol. 2005;20:323-329.

6. Callahan RJ, Chilton HM, Ponto JA, Royal HD, Bruce AD. Procedure guideline for the use of radiopharmaceuticals 4.0. J Nucl Med Technol. 2007;35:272-275.

7. Safety Guide for Radiation Protection in Nuclear Medicine. Vol 14.2. Yallambie, Australia: Australian Radiation Protection and Nuclear Safety Agency; 2008.

8. Applegate K. Pregnancy screening of adolescents and women before radiologic testing: does radiology need a national guideline? J Am Coll Radiol. 2007;4:533-536.

9. ACR Practice Guideline for Imaging Pregnant or Potentially Pregnant Adolescents and Women with Ionizing Radiation. Reston, VA: American College of Radiology; 2008.

10. Schreiner-Karoussou A. A preliminary study of issues and practices concerning pregnancy and ionising radiation. Radiat Prot Dosimetry. 2009;135:79-82.

11. Wilcox AJ, Baird D, Dunson D, McChesney R, Weinberg C. Natural limits of pregnancy testing in relation to the expected menstrual period. JAMA. 2001;286: 1759-1761.

12. Singh S, Darroch JE. Adolescent pregnancy and childbearing: levels and trends in developed countries. Fam Plann Perspect. 2000;32:14-23.

13. Chen XK, Wen SW, Fleming N, Demissie K, Rhoads GG, Walker M. Teenage pregnancy and adverse birth outcomes: a large population based retrospective cohort study. Int J Epidemiol. 2007;36:368-373.

14. Accreditation Board Regulations. Victoria, Australia: Australian and New Zealand Society of Nuclear Medicine; 2008.

15. Cole LA, Khanlian SA, Sutton JM, et al. Accuracy of home pregnancy tests at the time of missed menses. Am J Obstet Gynecol. 2004;190:100-105.

16. Bastian LA, Nanda K, Hasselblad V, Simel D. Diagnostic efficiency of home pregnancy test kits: a meta-analysis. Arch Fam Med. 1998;7:465-469.

17. Stenman UH, Tiitinen A, Alfthan H, Valmu L. The classification, functions and clinical use of different isoforms of HCG. Hum Reprod Update. 2006;12:769-784. 